\title{
Musique scolaire et société dans la France de la Troisième République
}

Michèle Alten

\section{(2) OpenEdition}

1 Journals

Édition électronique

URL : http://journals.openedition.org/trema/310

DOI : $10.4000 /$ trema.310

ISSN : 2107-0997

Éditeur

Faculté d'Éducation de l'université de Montpellier

\section{Édition imprimée}

Date de publication : 1 décembre 2005

Pagination : 5-19

ISSN : 1167-315X

\section{Référence électronique}

Michèle Alten, « Musique scolaire et société dans la France de la Troisième République », Tréma [En ligne], 25 | 2005, mis en ligne le 06 janvier 2010, consulté le 01 mai 2019. URL : http:// journals.openedition.org/trema/310 ; DOI : 10.4000/trema.310

Ce document a été généré automatiquement le 1 mai 2019.

Trema 


\title{
Musique scolaire et société dans la France de la Troisième République
}

\author{
Michèle Alten
}

1 Pour rendre compte de la situation de la musique dans l'institution éducative française, il est nécessaire d'étudier les différents facteurs qui ont conditionné, depuis la fin du XIXe siècle, son évolution. En effet, depuis 1880, l'enseignement musical scolaire est soumis à l'influence d'une double dynamique, à la fois externe et interne.

2 Les attentes exprimées par la société vis-à-vis de son école se traduisent en premier lieu par le choix des disciplines jugées utiles aux différents publics scolaires. Dans le bagage considéré comme nécessaire, la musique ne figure pas toujours et l'élite masculine du pays est longtemps privée d'enseignement musical. En second lieu, au-delà des différences d'éducation liées au sexe, l'évolution de la sensibilité politique nationale conduit, au XXe siècle, à envisager sous une autre forme la mission éducative et à privilégier la connaissance de l'enfant et son développement spécifique. La place de la musique apparaît alors sous un jour nouveau pour les responsables éducatifs et pour les maîtres.

Enfin, à l'intérieur même de la corporation enseignante, des aspirations et des modèles pédagogiques divergents entraînent des pratiques diversifiées et provoquent des affrontements et des débats dont l'écho assourdi est encore perceptible aujourd'hui.

4 Nous tenterons donc de mettre en évidence la pluralité des éléments qui sont entrés en interaction pour définir et faire évoluer, durant plus d'un demi-siècle, l'enseignement musical scolaire. Un refus des causalités univoques, manichéennes, souvent trop simples, nous semble indispensable à une compréhension réelle des évolutions. Celle-ci passe par un refus des images d'Epinal et des nostalgies et implique une lucidité qui permet seule de fonder une identité professionnelle tournée vers l'avenir. 


\section{Un enseignement réservé aux filles et aux enfants du peuple}

5 L'enseignement public français fonctionne, jusqu'à la Seconde Guerre mondiale, sur le principe de la séparation des publics scolaires. Les enfants du peuple fréquentent l'école primaire, les couches moyennes le primaire supérieur ou le collège et la bourgeoisie le lycée, des petites classes au baccalauréat. Dans ce schéma, la place de la musique varie selon le sexe et le type d'enseignement. Si le primaire et le primaire supérieur ne font pas de différence entre les sexes, les collèges et les lycées, qui reproduisent les clivages éducatifs des couches sociales aisées, réservent la musique aux jeunes filles. Après les années vingt, où le rapprochement des scolarités entre filles et garçons menace l'existence même de la musique, un tournant décisif s'opère en 1938, lorsque Jean ZAY décide de faire figurer la musique dans l'éducation masculine secondaire.

\section{I.1. L'éducation de la sensibilité féminine}

Lorsque Camille SEE fonde, en 1880, les lycées de jeunes filles, les besoins spécifiques d'un enseignement féminin sont l'objet d'un vaste débat. Pour ce qui concerne la musique, les discussions se focalisent autour de l'enseignement du piano, symbole de l'éducation bourgeoise. Celui-ci est violemment attaqué, parce qu'il contraint les filles à gaspiller à la fois leur temps, leurs forces et leur santé.

7 Charles GOUNOD, compositeur soucieux de progrès social, déclare en 1887: «Vous me demandez la part qu'il convient de faire à l'étude du piano dans l'éducation des jeunes filles? La réponse me parait des plus simples: le moins de temps possible pour celles qui ne doivent pas en faire leur profession. Voilà mon sentiment tout cru; je vous le livre. ${ }^{1}$

8 Dans cette hostilité au piano se rejoignent deux conceptions antagonistes de l'éducation féminine : ceux qui souhaitent rendre celle-ci moins frivole et plus intellectuelle et ceux qui veulent la rendre plus utilitaire et plus pratique. Quelques voix, attachées au modèle de féminité en vigueur dans les classes dominantes, déplorent, à l'inverse, l'excessif intellectualisme des programmes de lycée et réclament davantage de langues vivantes, de musique, de dessin et même de danse.

9 Ces divergences de points de vue, exprimées par des grandes figures intellectuelles ou politiques masculines, expliquent les remaniements successifs subis par les programmes d'enseignement secondaire féminin à la fin du XIXe siècle. Les projets initiaux envisagent deux heures de musique vocale obligatoire pendant les trois premières années de scolarité et une heure facultative pendant les deux dernières années. Mais après le passage en commission, dans les textes publiés en 1882 une heure de musique disparaît en troisième année pour permettre l'introduction d'un enseignement d'hygiène et d'économie familiale, la mission de future femme d'intérieur ne pouvant être négligée. Le sacrifice horaire est demandé à la musique, considérée comme art d'agrément, alors que le dessin garde ses trois heures hebdomadaires durant les trois premières années d'études. En 1897 les partisans d'un renforcement utilitaire et anti-intellectuel des programmes obtiennent une nouvelle victoire : deux heures de couture et une heure et demie de gymnastique sont introduites de manière obligatoire dans le cursus des trois premières années. On rogne alors une nouvelle fois sur la musique, qui se réduit 
désormais à une heure de solfège obligatoire. Malgré la nouvelle appellation qui le désigne, le contenu de cet enseignement musical reste très vague pour les décideurs, la musique étant la seule matière pour laquelle aucune indication de programme n'est fournie dans les textes officiels. Cependant le ministère, conscient de la faible qualification des maîtresses de chant, décide en 1889 d'encourager leur formation en titularisant celles qui auront réussi le certificat d'aptitude à l'enseignement du chant créé pour les écoles normales d'instituteurs et les écoles primaires supérieures.

10 Pour les jeunes filles aisées, destinées à devenir des épouses et des mères capables d'échanger avec leur mari et leurs enfants, l'enseignement de la musique constitue une reconnaissance de la spécificité affective et émotionnelle de leur sexe. Mais au XXe siècle les jeunes bourgeoises aspirent de plus en plus à passer le baccalauréat et à s'inscrire à l'université. Les scolarités féminine et masculine doivent alors se rapprocher. En 1928, le second cycle féminin s'allonge et s'intellectualise, faisant disparaitre les heures facultatives de musique qui avaient perduré jusque là. A cette date, le modèle masculin de culture scolaire semble, pour les élites, destiné à triompher.

\section{I.2. La musique pour le peuple}

11 Après une première apparition dans les programmes officiels nationaux de l'enseignement primaire en 1834 et 1836, le chant devient, le 27 juillet 1882, dans les textes promulgués par Jules FERRY, la douzième et dernière matière obligatoire de l'éducation intellectuelle. Désireux d'installer durablement un régime encore fragile, les républicains au pouvoir considèrent l'éducation comme un levier politique majeur, destiné à façonner une nouvelle communauté de conscience, à la fois intellectuelle, spirituelle et morale. La musique peut alors, à l'instar de ce qui se passe dans les pays protestants, contribuer à rassembler les citoyens autour d'un nouveau socle de valeurs. En effet, comme l'explique le scientifique républicain Albert DUPAIGNE, le contact des élèves avec l'art musical aura un double effet : d'abord la relation avec l'art élargira leur champ d'expérience, enrichissant ainsi leur intelligence et les rendant plus aptes à l'étude; ensuite l'apprentissage de textes de chants possédant une valeur littéraire et morale développera leur sens du devoir, leur amour de la patrie, de la famille et de la nature ${ }^{2}$.

12 Mais cette ambition se heurte à un obstacle : les ouvrages pédagogiques et les guides d'instituteurs en cours avant 1882 ne font aucune mention des mots chant et musique. L'absence de tradition, de méthode et de répertoire spécifiques à destination des écoles (nous laissons ici de côté le cas particulier des intervenants musiciens municipaux parisiens) conduit donc le législateur à inventer de toutes pièces un programme d'activités destiné aux différents niveaux, "laborieux échafaudage élevé en dehors des indications fournies par l'expérience $»^{3}$, selon les termes de l'inspecteur d'académie de la Marne CORNET.

13 Les textes de 1882 juxtaposent des activités de chant et des enseignements de solfège. De la section enfantine (5 à 7 ans) jusqu'au cours supérieur (11 à 13 ans) les chants, à une ou deux voix, doivent être appris par l'audition. Après apprentissage, les chants doivent être utilisés pour imprimer bonne humeur et discipline aux entrées et aux sorties de classe. L'instituteur doit donc se transformer régulièrement en chanteur pour parvenir à faire acquérir un bagage de chants aux élèves. En plus de cette activité vocale, l'exigence officielle concerne également les éléments fondamentaux du solfège. Au cours 
élémentaire les programmes se limitent à la reconnaissance des notes, mais au cours supérieur la connaissance de la clé de sol, de la clé de fa, de la gamme diatonique majeure, des intervalles naturels, des signes altératifs et des principaux tons majeurs et mineurs est demandée. Le volume horaire prévu pour réaliser cette ambition est de une à deux heures par semaine. En 1923 les programmes restent quasiment identiques, tout en affirmant la nécessité d'une éducation de la voix et de l'oreille comme préalable à toute étude théorique de la musique. L'horaire est réduit à une heure. En 1938 enfin une directive de Jean ZAY, ministre du Front Populaire, fait entrer la musique dans un bloc de trois heures qui l'assimile à des activités dirigées destinées à l'ouverture culturelle et pédagogique, sans que soient modifiés les contenus de programmes. Jusqu'à sa disparition en 1941, le primaire supérieur féminin et masculin, voie royale pour la préparation au concours des écoles normales d'institutrices et d'instituteurs, bénéficie, pour sa part, d'un traitement relativement privilégié, avec des enseignants recrutés sur concours et des horaires de cours stables durant les quatre années de scolarité (une heure de chant obligatoire par semaine et une heure facultative de renforcement dans les trois dernières années).

Durant la Troisième République, si la stabilité des instructions officielles concernant la musique dans l'enseignement primaire traduit la permanence d'une volonté politique affichée, les conditions de la mise en oeuvre des programmes prévus restent difficiles. L'alphabétisation requiert souvent toute l'énergie des maîtres - le musicologue Julien TIERSOT reconnait, en 1911, que peu d'instituteurs sont capables d'ajouter à leurs fatigues ordinaires l'effort exceptionnel et parfois épuisant de l'apprentissage du chant ${ }^{4}$ - et la préparation du Certificat d'études apparaît, après la Première Guerre mondiale, comme un enjeu majeur pour l'avenir de tous les enfants qui arrêtent leur scolarité à la fin du primaire, c'est-à-dire pour les quatre cinquièmes des élèves français.

\section{I.3. L'introduction tardive de la musique dans les lycées de garçons}

Les premières années de scolarité du jeune bourgeois dans les petites classes du lycée ne comportent, jusqu'en 1902, aucune heure d'enseignement consacrée à la musique. Destinées à préparer l'entrée dans l'enseignement secondaire, ces trois années d'études ne constituent qu'une longue propédeutique, autour des pôles disciplinaires ultérieurs. Mais en 1902 le modèle pédagogique élaboré pour l'enseignement primaire ne peut plus être ignoré et il inspire un profond remaniement des petites classes. Une année supplémentaire voit le jour. La liste des matières change et s'allonge. Les libellés deviennent précis (calcul, leçon de choses, petits récits historiques...) et le chant fait son apparition, à raison d'une heure par semaine, dans chacun des niveaux de classe. Cependant, à la différence de l'enseignement primaire, nulle trace ici de contenu de programme pour cette nouvelle matière (alors que le dessin bénéficie d'instructions très détaillées sur le type de dessin à effectuer). Jusqu'à leur disparition officielle en 1926, les petites classes des lycées cultivent donc le paradoxe d'un enseignement de musique obligatoire mais sans indication de contenu.

Durant les quatre années de premier cycle secondaire masculin, la musique reste absente jusqu'en 1938. A l'inverse, le dessin, qui avait disparu en 1884, réapparait dans les textes officiels de 1902 et se renforce dans ceux de 1926 à travers la création d'un nouvel enseignement d'histoire de l'art. L'année 1938 constitue donc une évolution majeure pour la musique. Filles et garçons sont traités sur un pied d'égalité et bénéficient, durant 
quatre ans, d'une heure de musique par semaine. Cette mutation s'inscrit dans une réactualisation d'ensemble des finalités de l'enseignement secondaire. Une culture générale bien comprise se doit de développer à la fois l'intelligence, le cœur, le caractère, le sens moral et le goût du beau. Le programme proposé aux enseignants comporte quatre rubriques : le travail de la voix, l'apprentissage d'un répertoire vocal, une initiation à la théorie musicale et un bagage culturel en histoire de la musique. Le programme de troisième se termine par la découverte de l'accord de septième de dominante et par l'interprétation de chœurs à plusieurs voix. Les compositeurs à faire découvrir aux élèves sont laissés au choix du professeur, dans une période allant de Lulli à nos jours. Les nouveaux moyens de reproduction du son doivent permettre une découverte sonore des oeuvres. Ainsi les jeunes gens des deux sexes pourront acquérir une égale culture musicale. Cependant Jean ZAY ne sous-estime pas la difficulté de la tâche, conscient de la forte disparité de niveau des élèves dans ce domaine.

Dans les trois classes de second cycle, les programmes privilégient, jusqu'en 1902, exclusivement l'héritage des humanités classiques. En 1902 la création de sections scientifiques favorise la présence du dessin, mais non de la musique. La demi-heure d'art introduite de manière facultative en 1926, puis obligatoire en 1931, ne concerne que la peinture, la sculpture et l'architecture. La dimension patrimoniale de l'œuvre musicale, à la trace éphémère, est matériellement encore difficile à prendre en compte.

Une certaine idée de la virilité, hostile à ce qui semble appartenir au registre de la sensibilité ou de l'expression collective, conduit l'enseignement des lycées de garçons à ignorer complètement, en 1880, la musique. Le rapprochement progressif des modèles éducatifs conduit le législateur à privilégier la dimension universaliste de la culture aux dépens des particularismes sociaux et sexuels.

19 Très marqués par l'héritage révolutionnaire de 1789 , les hommes politiques de la Troisième République font de la rationalité intellectuelle la base de l'éducation du citoyen. A ce titre ils se méfient, à l'instar de Condorcet, des débordements suscités par les émotions collectives. La musique apparaît alors suspecte, voire dangereuse. Mais en même temps, ils reconnaissent son pouvoir comme instrument au service d'une sacralité laïque et comme moyen d'expression de la sensibilité. Cette ambivalence dans le rapport à la musique explique, pour une large part, la fragilité de cette discipline dans l'institution scolaire.

\section{Un enseignement lié aux évolutions de la culture scolaire}

Les mutations politiques, scientifiques et techniques du premier vingtième siècle rejaillissent fortement sur l'école primaire, sur ses valeurs, ses comportements et ses pratiques. Le regard porté sur l'école et sur l'enfant change et cette modification entraîne 
une évolution sensible des manières d'être et de faire. La musique participe de ce phénomène, à travers une profonde mutation de son statut et de son rôle dans l'école.

\section{II.1. La musique auxiliaire de l'instruction civique}

21 La France républicaine de 1882 donne à l'école publique un rôle majeur dans le processus d'adhésion de tous à la nation. Presque toutes les matières d'enseignement concourent à édifier cette nouvelle identité. Dans les différents manuels de lecture, de récitation, de grammaire et de chant, composés de morceaux choisis, se retrouvent la même thématique à la gloire de la patrie. Les Chants patriotiques de Déroulède sont mentionnés dans 50 listes départementales d'ouvrages autorisés en 1885. Le premier recueil signé BOUCHOR et TIERSOT et publié en 1895 contient certains morceaux qui incitent à l'action et au combat armé pour la patrie. Mais au début du XXe siècle, la guerre de 1870 s'est éloignée et l'inspiration nationaliste fait place à un humanisme républicain soucieux de progrès social et de paix. Le troisième volume des Chants populaires pour les écoles (BOUCHOR-TIERSOT) ne contient plus de chants à la thématique revancharde et le recueil de Déroulède disparait de la plupart des listes départementales en $1909^{5}$.

le progrès social annoncé ne signifie pas remise en cause de l'ordre établi. Si la République est le seul régime capable d'engendrer une dynamique de libération de tous, c'est d'abord l'acceptation de son sort et le courage au travail qui conditionnent le progrès collectif. Les devoirs de chacun sont à la fois individuels, domestiques et sociaux. En 1886, Claude Augé chante l'écolière studieuse et le jeune montagnard qui s'exile pour trouver du travail, tandis que Maurice BOUCHOR, en 1907, loue le courage et l'ardeur au travail des verriers et des tisserands.

Les orientations contenues dans les manuels trouvent un écho favorable chez les instituteurs formés dans le moule républicain des écoles normales. Mais la lourdeur des programmes d'études (16 matières obligatoires dans le cursus du normalien) ainsi que la qualité inégale d'une formation musicale assurée par des professeurs spéciaux (ce sont des intervenants extérieurs qui enseignent "les matières accessoires ») constituent un frein au développement des pratiques. Néanmoins les témoignages d'instituteurs et d'élèves sur l'école d'avant 1914 se rejoignent pour attester de l'apprentissage de chants patriotiques dans la France des bourgs et des campagnes. Dans l'Eure-et-Loir, dans les Deux-Sèvres, en Saône-et-Loire et dans la Manche se retrouve le même répertoire. Les spécificités politiques et culturelles régionales introduisent toutefois quelques différences. Dans les régions où la présence de l'église catholique est très forte, les chants affirment d'abord la laïcité républicaine. Dans celles de la France du Sud où l'alphabétisation est faible, les priorités sont données à l'écriture et à la lecture; l'enseignement musical est inexistant. A l'inverse, dans une zone préalablement alphabétisée, un instituteur quelque peu musicien, tel Marc VILLIN à Saint-Barthélémy en Seine-et-Oise, peut faire apprendre les divers morceaux des Chants de l'enfance de Claude AUGE à ses élèves de classe unique.

Face à l'injonction musico-civique du législateur, les maîtres réagissent donc à la fois en fonction des urgences du terrain et de leur bagage artistique. Cependant, la mémorisation de quelques « succès » du répertoire scolaire patriotique (Où t'en vas-tu soldat de France?) constitue un socle musical commun, qui vient renforcer le message transmis par les autres disciplines scolaires. 


\section{II.2. Le souci de la spécificité enfantine} des XIXe et XXe siècles, un répertoire folklorique régional menacé par le succès des modèles musicaux urbains. Julien TIERSOT, Louis BOURGAULT-DUCOUDRAY, Vincent d'INDY, Charles BORDES, Déodat DE SEVERAC et Maurice EMMANUEL publient des recueils qui font revivre ce patrimoine. Si les chants populaires traditionnels incarnent les traits essentiels du génie de la collectivité nationale, il faut à tout prix les empêcher de mourir et les transmettre aux générations futures. En 1923, le XIe congrès de la Société française de l'art à l'école, consacré au chant et à la musique, émet le vœu que soit éditée une anthologie du chant scolaire fondée sur le folklore régional ; en 1925-1926, l'éditeur HEUGEL publie dix fascicules consacrés au folklore de dix provinces françaises. La hiérarchie éducative supérieure soutient cette initiative. L'inspecteur général Charles L'HOPITAL consacre au sujet un long développement dans une conférence prononcée en Sorbonne en $1933^{6}$. Pour lui, la chanson populaire traditionnelle constitue la compagne idéale de l'enfance, car elle est l'expression simple et naïve du peuple, dans sa franchise, sa joie, son ironie, sa fantaisie et son imagination. Son collègue l'inspecteur général Oscar AURIAC partage son point de vue et conseille en ce sens les instituteurs qu'il visite dans le Sud-Ouest de la France entre 1930 et 1935. Mais les inspecteurs de l'enseignement primaire, en contact permanent avec les classes, expriment un avis plus nuancé sur le sujet. Ils savent en effet que les chants de terroir sont devenus des documents ethnographiques et historiques qui n'éveillent plus aucun écho ni chez les instituteurs ni chez les élèves et que leur apprentissage apparaît alors bien factice ${ }^{7}$.

La chanson enfantine constitue l'autre alternative au répertoire civique discrédité. Une floraison de nouveaux manuels est proposée aux enseignants de l'entre-deux-guerres. Certains, peu nombreux, gardent une facture classique et sont destinés à développer l'expression vocale. Mais la majorité d'entre eux opère une rupture profonde avec le passé en associant étroitement la dimension corporelle à l'activité chantée. L'influence des recueils publiés en Suisse par Emile JAQUES-DALCROZE avant 1914, qui privilégient la dimension rythmique comme base de l'éducation musicale et corporelle, se fait directement sentir dans certains ouvrages. L'éducation physique est alors convoquée 
pour apporter son concours à des chants conçus pour être dansés sur des rythmes de marche, de ronde, de mazurka, de gavotte ou de menuet. Toutefois les manuels les plus nombreux privilégient la dimension expressive et ludique de l'activité corporelle, à travers la mise en scène de saynètes chantées et mimées. Deux auteurs se partagent l'essentiel des titres: le parolier Hermin DUBUS et le compositeur Paul SCHLOSSER, souvent associés dans des recueils communs. Les revues pédagogiques et syndicales ( l'Ecole libératrice) fournissent également des choix de chants qui incitent les enseignants à célébrer les animaux, les paysages, les saisons, les métiers, la famille et les fêtes. Quant aux trente-deux pratiques de classe de l'entre-deux-guerres retrouvées grâce aux cahiers de chant et de récitation du Musée pédagogique de Rouen, elles confirment les évolutions mises en évidence dans les livres scolaires et les revues pédagogiques. Trois types de chants sont présents : les célébrations de la nature, le folklore traditionnel et les chansons enfantines.

Le chant scolaire a définitivement cessé de servir d'auxiliaire à l'édification citoyenne et nationale dont la République fragile et menacée avait besoin pour assurer sa pérennité.

\section{II.3. La culture musicale à portée de tous} responsables éducatifs voient dans les progrès techniques liés à la reproduction du son une solution pour pallier les carences de formation des maîtres. Deux appareils leur semblent pleins de promesses: le phonographe et la radio. Malgré leur coût et leur imperfection technique, de nombreux instituteurs, stimulés par l'enthousiasme de leur syndicat pour ces nouvelles découvertes, se lancent, dans les années trente, dans l'aventure.

31 Dès 1925 la maison Pathé propose deux disques contenant chacun deux chansons scolaires. En 1929, une campagne associe Pathé, le ministère de l'Instruction publique et le Comité français du phonographe dans l'enseignement. Elle offre cent phonographes et cent séries de disques à des écoles normales primaires. Le Comité met progressivement en place une logistique qui permet de sélectionner les disques, de rédiger un commentaire pédagogique et d'obtenir des prix d'achat avantageux. Mais le prix d'un phonographe reste élevé pour des municipalités qui n'en perçoivent pas toujours l'intérêt. Devant la difficulté, certaines écoles s'organisent. Dans l'Ariège un inspecteur primaire organise une circulation des phonographes et des disques afin de faire accéder toutes les écoles de sa circonscription " aux plaisirs des régions riches $»^{8}$. En Haute-Vienne une discothèque de prêt est mise sur pied. Les initiatives d'équipement dépassent souvent le cadre de l'enseignement, grâce à la mobilisation des amicales d'anciens élèves et des coopératives scolaires. Mais la faible adaptation pédagogique de l'outil explique le transfert des attentes de progrès, à la fin des années vingt, vers l'autre découverte majeure dans le domaine du son : la TSF.

Jusqu'en 1929, la radio en est à ses balbutiements. Le poste parisien de Radio Tour Eiffel appartient à l'armée qui prête une partie de son temps d'antenne pour des émissions publiques telles les leçons du Collège de France ou les cours de la Sorbonne. A partir du 12 octobre 1929, un accord signé entre Radio Tour Eiffel et le SNI permet l'organisation hebdomadaire d'une émission intitulée "L'heure radiophonique de l'école ». Après une interruption pour travaux, l'émission reprend le 21 janvier 1933. Elle dure une demiheure et comporte deux rubriques musicales (un chant et une séquence d'histoire de la 
musique) sur les cinq proposées. Mais les départements éloignés de Paris ne peuvent la capter et ils doivent imaginer localement des émissions sur les postes régionaux. Le plus exemplaire d'entre eux est sans conteste la Gironde, dans laquelle les instituteurs organisent une heure hebdomadaire d'émission presque exclusivement consacrée à la musique (sept rubriques sur neuf). Le programme comporte des extraits d'œuvres classiques françaises qui vont de l'opérette à la tragédie lyrique, une grande variété de chants scolaires ainsi qu'une tentative d'apprentissage radiophonique intitulée "Nous apprenons à chanter ». Celle-ci, particulièrement novatrice, regroupe dans un studio des élèves qui, au fil des séances, reçoivent des consignes et exécutent en direct ce qui leur est demandé. Le maître dans sa classe peut se caler directement sur la progression proposée. Fort de toutes les initiatives locales de ses membres, le SNI élabore, en 1932, un ambitieux programme de radiophonie scolaire qui associe la radio, le disque et le support papier dans une synergie nouvelle. Mais ses propositions ne sont que très partiellement reprises par le gouvernement de Front populaire lorsque celui-ci réorganise au plan national les émissions de radio scolaire. En 1937, deux courtes séquences musicales sont diffusées chaque semaine sur l'émetteur de Paris PTT : une écoute commentée de disque classique et en alternance, soit une initiation musicale, soit un apprentissage de chants. Lorsque, en 1938, la tension politique internationale s'accroit, les émissions scolaires disparaissent provisoirement des grilles de programmes. Réapparues en novembre 1938, elles réduisent désormais la musique à vingt minutes de chant choral ${ }^{9}$.

Malgré les difficultés économiques de la période, l'école élémentaire française adopte progressivement, dans l'entre-deux-guerres, un nouveau modèle de scolarisation, fondée sur l'attention portée à l'enfant et sur l'ouverture culturelle. La musique participe de ce mouvement à travers un profond renouvellement de ses formes d'activités.

\section{Un enseignement divers dans ses exigences et ses pratiques}

Deux univers se partagent l'enseignement musical public : celui des lycées de jeunes filles qui vit en étroite relation avec la culture musicale des milieux dominants et celui des écoles, traversé de prises de position pédagogiques et d'influences culturelles multiples. A un monde de stabilité s'oppose donc un monde de débats et de mouvement.

\section{III.1. La tradition musicale des lycées de jeunes filles}

Si l'absence totale de programme livre les professeurs à eux-mêmes et ne permet pas de fixer un niveau d'exigence minimum commun qui crédibiliserait la discipline, les établissements publics secondaires féminins donnent cependant à la musique une place éminente lors des deux grands types d'événements qui manifestent leur ouverture sur le monde : les fêtes de charité et les manifestations commémoratives. En province comme à Paris les jeunes filles sont alors mobilisées pour jouer d'un instrument et pour chanter. En 1893, les lycéennes de Lyon donnent un concert de charité. En 1908, le lycée de Nantes fête ses vingt-cinq ans dans une fête où les élèves les plus âgées se produisent dans un orchestre à cordes tandis que les plus jeunes chantent et dansent des gavottes et des menuets en costumes. En 1909 le lycée FENELON, à Paris, fête lui-aussi ses vingt-cinq ans dans une imposante cérémonie. La grande et la petite chorale réunies sont dirigées par Gabriel PIERNE, compositeur prix de Rome, dans des œuvres françaises de SAINT-SAËNS, 
FRANCK et PIERNE. A partir de 1907, grâce à la détermination de l'inspecteur parisien Jules COMBARIEU, sont créés dans tous les lycées de filles parisiens, des ensembles vocaux. Ceux-ci utilisent une méthode unique d'apprentissage du chant, intuitive, directe, sans théorie ni solfège, mise au point par COMBARIEU lui-même et imposée par arrêté ministériel. Malgré la présence d'une activité chorale importante, l'absence de programme officiel, en particulier en histoire de la musique, est considérée comme une lacune grave et dénoncée à plusieurs reprises, par COMBARIEU d'abord en 1914, par PIERNE ensuite en $1917^{10}$

Durant les années vingt, la situation n'évolue guère. A l'inverse, durant les années trente, les militants de la toute nouvelle Union française des oeuvres laïques d'éducation artistique, dénonçant le chômage massif qui frappe les artistes, se mobilisent pour dynamiser l'enseignement musical secondaire. De 1936 à 1940, des concours de musique instrumentale et de chant individuel sont organisés au plan national, avec un programme d'œuvres classiques ambitieux ainsi que des concours de composition de chansons. Les professeurs, qui obtiennent, à partir de 1938, une reconnaissance accrue, fondée sur un programme commun aux deux sexes, se sentent partie prenante de l'édification d'une culture générale humaniste, imaginée à partir d'un patrimoine d'œuvres littéraires, scientifiques et artistiques constitutives de la formation générale des enfants de la bourgeoisie.

La stabilité sociale et culturelle du recrutement des lycées jusqu'à la moitié des années cinquante va permettre au modèle d'activités musicales largement hérité du XIXe siècle de perdurer au-delà de la Seconde Guerre mondiale. La remise des carnets de notes, les distributions de prix, les participations à des cérémonies locales diverses ainsi qu'à des concours de chant choral sont autant d'occasions, dans les années cinquante, de faire vivre la musique lycéenne.

\section{III.2. Le conflit pédagogique dans les écoles}

En 1882, les programmes relatifs à la musique restent muets sur la question des méthodes d'apprentissage à employer dans les classes. Cependant, la sous-commission chargée de la rédaction des textes émet le désir de voir expérimenter comparativement dans les écoles de la Seine deux méthodes différentes, la méthode usuelle inspirée des conservatoires et la méthode GALIN-CHEVE, spécialement conçue pour les débutants non musiciens. Les expériences menées dans douze écoles primaires parisiennes en 1887 concluent à la validité de la méthode alternative, dite «chiffrée» dans la majorité des exercices demandés. Dénonçant le poids de la routine et des préjugés, un certain nombre de pédagogues se mobilisent pour faire connaître ce succès, fondé sur l'écoute des sons, le repérage des intervalles et la suppression de la tonalité absolue. Ils obtiennent, en 1905, que les normaliens reçoivent, durant leur formation, une information sur le sujet et gagnent à leur cause la principale revue pédagogique de l'époque, le Manuel général de l'instruction primaire. Sans remettre en cause le bien-fondé de la notation classique pour les élèves qui se destinent à une formation musicale poussée, ils plaident pour une simplicité capable de donner à tous les rudiments constitutifs d'une vulgarisation de l'art musical ${ }^{11}$.

Face à cet enthousiasme vulgarisateur, les professeurs spéciaux de la ville de Paris, recrutés sur des compétences de musiciens classiques, se sentent menacés dans leur légitimité et s'unissent, à partir de 1912, pour organiser la contre-attaque. Dans des 
articles polémiques, ils dénoncent le coup de bluff et l'absurdité de la méthode. Ils en appellent au soutien de compositeurs éminents (SAINT-SAËNS, D'INDY) ainsi qu'à l'ensemble des enseignants de musique. Mais cette attitude purement défensive reste inefficace et il faut l'intervention d'un jeune professeur de la ville de Paris, Maurice CHEVAIS, pour que la réflexion prenne, à partir de 1914, un tour plus constructif. Celui-ci reconnaît le caractère louable de la volonté simplificatrice des galinistes. Il va donc s'en inspirer pour proposer, en 1920, un programme musical de base fondé sur l'écriture musicale usuelle. Il limite les ambitions solfégiques à la gamme de do majeur et à la clé de sol, admet la pertinence de la reconnaissance des sons et des intervalles comme préalable à l'écriture des signes et reconnaît l'importance du chant comme base et aboutissement de l'enseignement musical. Le Conseil supérieur de l'instruction publique, chargé de la rédaction de nouveaux programmes, tient compte de cette réflexion ainsi que de l'expérience menée par un célèbre professeur du conservatoire de Paris, André GEDALGE, dans une école de campagne. Celui-ci a développé chez les enfants la capacité à entendre intérieurement les intervalles et est ainsi parvenu, en deux ans, à faire d'eux des musiciens. Malgré le caractère très particulier de l'expérience, l'affaire semble donc entendue et le galinisme disparaît des textes officiels en 1923.

Le plus difficile reste alors à faire: proposer à tous les instituteurs une méthode suffisamment claire et graduée pour leur permettre de l'appliquer dans leur classe. Si A. GEDALGE fait paraître le résultat de son expérience dans un ouvrage intitulé L'enseignement de la musique par l'éducation méthodique de l'oreille, c'est surtout Maurice CHEVAIS qui devient, dans les différentes revues destinées aux instituteurs, le pédagogue inlassable de la musique. Dans le Manuel général il publie, à deux reprises, deux longues séries d'articles. En 1923-24, il développe, à raison d'une leçon par mois, les différentes étapes de la formation de l'oreille, en commençant par l'intervalle de quinte do-sol. Chaque leçon comporte huit rubriques: écoute active de chant, pratique de chant, identification active de sons écrits au tableau, chant de ceux-ci, identification de formules mélodiques créées à partir des mêmes sons, reproduction de celles-ci grâce à un appui mental sur les sons de l'accord parfait, travail d'audition intérieure et enfin apprentissage d'un chant d'application. En 1924-25, il passe à l'étude des rythmes, toujours à raison d'une leçon par mois. Ces deux cycles s'adressent au cours élémentaire. En 1925-26, il s'adresse au cours moyen en quatre leçons autour des signes musicaux et d'exercices fondés sur les marches harmoniques, se gardant bien d'aborder les changements de tonalité prévus théoriquement au programme. Il termine son cycle, en 1926-27, par une leçon sur la polyphonie destinée au cours supérieur. Au cours des années trente, il reprend son propos, dans le Manuel général d'abord, dans l'Ecole libératrice ensuite, dans une publication en trois volumes enfin, intitulée Éducation musicale de l'enfance. Ainsi jusqu'à sa mort en 1942, Maurice Chevais incarne une volonté de concilier exigence et réalisme, dans son combat sans relâche au service de la musique scolaire.

\section{III.3. Des formes imprévues de pratique musicale}

41 Si les professeurs spéciaux maintiennent à Paris des pratiques d'enseignement en adéquation avec les textes officiels, des initiatives novatrices, aucunement prescrites par le législateur, voient le jour dans un grand nombre d'écoles des années trente. Les nouvelles activités, centrées sur la réalisation de spectacles musicaux, ont leur origine dans la mobilisation des instituteurs. Sollicités, à partir de 1915, pour venir en aide aux enfants victimes de la guerre, ceux-ci deviennent, dans l'entre-deux-guerres, des agents 
actifs de la solidarité nationale. La création de coopératives diverses leur permet de financer des équipements de base nécessaires au mieux-être matériel (cantines, lavabos) et pédagogique (sorties, équipement) des élèves. Tous les moyens sont imaginés pour récolter des fonds. Les écoles envisagent alors de s'adresser aux parents, en valorisant les compétences artistiques des enfants à travers des spectacles spécialement conçus pour populariser la nouvelle culture scolaire.

Dans les différentes fêtes scolaires qui sont alors organisées, la musique occupe une place prépondérante. Le concepteur des programmes de fêtes n'est d'ailleurs autre que Maurice CHEVAIS, devenu inspecteur du chant dans les écoles de Paris. A partir de 1927, il publie, à la demande des enseignants, de nombreuses suggestions dans le Manuel général puis dans l'Ecole libératrice. Les spectacles, qu'il propose sont conçus de manière thématique, autour de la célébration des rites calendaires (Noël, Pâques), des anniversaires républicains (14 juillet, 11 novembre, cinquantenaire de l'école laïque), des célébrations culturelles (Victor Hugo, romantisme, provinces de France) des permanences naturelles (les moments du jour, les saisons, les paysages) ou des activités humaines (le foyer, les voyages). Chaque thème est divisé en plusieurs rubriques et pour chacune d'entre elles un choix de musique classique, de chants, de poèmes, d'extraits de romans et de pièces de théâtre est offert. Par souci de réalisme, Chevais conseille aux maitres de se limiter à vingt numéros, soit une fête d'environ deux heures à deux heures et demie. Mais il sait bien que dans les préaux d'école les séances de quatre à cinq heures ne sont pas rares20. La préparation matérielle (décors, costumes) associe les enseignants, les parents et les anciens élèves. Lorsque ces derniers participent au spectacle, les ambitions culturelles de celui-ci s'en trouvent renforcées. Le vif succès obtenu par ce type de manifestation (600 personnes dans une école ouvrière du Lot-et-Garonne) assure aux organisateurs de substantiels bénéfices.

La préparation musicale des fêtes suppose un travail minutieux en amont, à la fois sur la justesse des voix, sur la qualité de l'interprétation et sur le respect des rythmes dans les déplacements dansés. Même si la nature des numéros varie en fonction de la compétence des maîtres et de la richesse des écoles (présence ou non d'un piano, d'un phonographe) l'enseignement de la musique prend ici un sens immédiat pour tous. Il devient un vecteur d'épanouissement de l'enfant, d'ouverture culturelle des parents et de valorisation de l'école au service d'une mission de solidarité. Placée en situation d'hégémonie culturelle sur un territoire encore faiblement touché par la culture de masse, l'école dépasse ici sa mission initiale d'instruction et tente de populariser un répertoire musical fait de rondes enfantines, de chant choral, de saynètes musicales et d'œuvres savantes. D'une manière non prévue par le législateur, la musique bénéficie alors d'une considération qui ne survivra guère aux bouleversements de l'après-guerre.

Sous la IIIe République, au-delà des divergences profondes qui traversent la pratique musicale scolaire, un dénominateur commun s'impose cependant à toutes les formes d'enseignement: la prééminence du chant. Il sacralise les rituels lycéens, fonde l'apprentissage du solfège et magnifie l'expression enfantine.

Discipline au statut ambigu, partagée entre le rationnel et l'émotionnel, faiblement reconnue par l'institution éducative, la musique scolaire peine, entre 1880 et 1939, à se dégager des images négatives qui lui sont associées. Elle doit se débarrasser de l'étiquette 
d'art d'agrément qui interdit son accès aux garçons des milieux privilégiés. Elle doit aussi s'affranchir du modèle parisien qui réserve son enseignement à des professeurs spécialistes. Elle doit enfin dépasser l'usage politique que les responsables républicains ont décidé de lui faire jouer. Les évolutions de la société française au XXe siècle, par le rapprochement des modèles scolaires entre filles et garçons, par le déclin du républicanisme militant dans les contenus d'enseignement et par l'engagement des enseignants dans une vision ouverte de la culture, favorisent le développement de nouvelles activités musicales. Cependant, à cause d'une formation initiale insuffisante ou inadaptée, à cause aussi du discours trop ambitieux ou inexistant du législateur, les enseignants se sentent souvent démunis pour aller au-delà du chant appris par l'audition. Des réussites exceptionnelles existent néanmoins. Elles tiennent toujours à une mobilisation particulière des acteurs de terrain (inspecteurs primaires, directeurs d'école, enseignants) qui font de l'apprentissage musical au plus grand nombre un sacerdoce. Mais leurs efforts sont peu reconnus. En effet l'atomisation du corps social, et l'isolement du citoyen face à l'Etat engendrés par la Révolution de 1789 nuisent au développement de pratiques culturelles communautaires et à leur pérennisation. Dans une société peu disposée à encourager l'ouverture de l'accès à la musique, l'école publique de l'entredeux-guerres tente, à sa façon, avec ses moyens, ses modèles et ses convictions, de jeter les bases d'une sociabilité et d'une culture musicales pour tous les élèves.

\section{NOTES}

1. L'enseignement secondaire des jeunes filles, Paris, Librairie Léopold Cerf, janvier-juin 1887, p. 145.

2. Rapports sur l'enseignement du chant dans les écoles primaires, Ministère de l'Instruction publique, 1881, pp. 67-89.

3. Cornet, «L'enseignement du chant ", Monographies pour l'exposition de 1889, 1889, p. 665.

4. Article "Chant», Nouveau dictionnaire de pédagogie et d'instruction primaire, Paris, Hachette, 1911, p. 249.

5. Alten, M., La musique dans l'école de J. Ferry à nos jours, Issy-les-Moulineaux, Ed. EAP, 1995, p. 84.

6. L'Hôpital, Ch., «Éducation musicale et enseignement du chant », Bulletin des personnels des Cours complémentaires, 1933, pp. 1-50.

7. Manuel général de l'instruction primaire, partie générale, année 1934-35, p. 570.

8. Auriac, O., L'école exemplaire. Initiatives et suggestions, Paris, A. Colin, 1948, p. 284.

9. L’Ecole libératrice, partie générale, année 1938-39, p. 100.

10. Pierné, G., Lettre du 26 mai 1917 à Pierre Lalo, Archives Vincent Laloy.

11. Manuel général de l'instruction primaire, partie générale, année 1903-1904, p. 543. 


\section{RÉSUMÉS}

Ce texte expose les processus et les difficultés inhérentes qui accompagnèrent l'émergence de l'éducation musicale. Michèle Alten relate l'histoire de la discipline depuis la fin du dix-neuvième siècle lorsque la musique devint en 1882 la douzième et dernière matière obligatoire enseignée à l'école. Son enseignement fut longtemps réservé aux seules jeunes filles et tarda à être généralisé sous la Troisième République.

This text deals with the processes and difficulties that came out with the beginning of music teaching. Michèle Alten recounts the history of music as a school subject from the seventeenth century, when it became the twelfth and last statutory subject at school. For a long time music teaching was reserved for young ladies and was not extended until late under the Third Republic.

\section{AUTEUR}

\section{MICHÈLE ALTEN}

Ingénieur de recherche habilitée, Université Paris-Sorbonne 